\title{
Estrés psicológico materno como posible factor de riesgo prenatal para el desarrollo de dificultades cognoscitivas: caracterización neuropsicológica de una muestra colombiana"
}

\author{
Psychological Maternal Stress as a Possible Prenatal Risk \\ Factor for the Development of Cognitive Problems: \\ Neuropsychological Characterization of a Colombian Sample
}

Recibido: enero 15 de 2010 | Revisado: marzo 20 de 2010 | Aceptado: abril 3 de 2010

\author{
María Cristina Pinto-Dussán ** \\ Oscar Mauricio Aguilar-Mejía \\ JUAN DANIEL GÓMEZ-ROJAS \\ Pontificia Universidad Javeriana, Bogotá, Colombia
}

Para citar este artículo. Pinto Dussán, M.C., Aguilar Mejía, O.M. \& Gómez Rojas, J.D. (2010). Estrés psicológico materno como posible factor de riesgo prenatal para el desarrollo de dificultades cognoscitivas: caracterización neuropsicológica de una muestra colombiana. Universitas Psychologica, 9 (3), 749-759.

* Artículo de investigación.

** Carrera 5 * \# 39-00, piso 2, oficina 218. Correos electrónicos: mcpduss@hotmail.com; oaguilar@ javeriana.edu.co; jd.gomez@javeriana.edu.co

\section{RESUMEN}

El estrés es una respuesta adaptativa que puede implicar cambios adversos a mediano y largo plazo. Investigaciones evidencian que el estrés en madres gestantes, puede tener diversos efectos sobre el desarrollo de los infantes. El objetivo del estudio fue describir las características cognitivas de menores cuyas madres reportan estrés durante el embarazo, como único factor de riesgo, en una población clínica de la ciudad de Bogotá. Se caracterizan los perfiles cognitivos, a partir de las evaluaciones neuropsicológicas, resaltando las puntuaciones significativas en los subtests e índices globales de la escala WISC IV. Se encontró que los niños cuyas madres sufrieron estrés durante el embarazo, presentan déficits en atención, funciones ejecutivas y velocidad de procesamiento.

Palabras clave autores

Estrés, embarazo, evaluación neuropsicológica, atención, funciones ejecutivas.

Palabras clave descriptor

Madres, psicología, pruebas neuropsicológicas, función ejecutiva.

\footnotetext{
A B S T R A C T

Stress is an adaptive response that may involve adverse changes in the medium and long term. Research showed that maternal stress during pregnancy may have variables effects on the offspring development. The objective of this study was to describe the cognitive characteristics of children from Bogota-Colombia, whose mothers during neuropsychological consultation, reported stress during pregnancy as the only risk factor. Cognitive profiles are described from neuropsychological assessments, highlighting significant scores in the subtest and global indices of the WISC IV scale. It was found that children whose mothers suffer stress during pregnancy have deficits in attention, executive functions and processing speed.

Keywords authors

Stress, Prenatal Risk, Cognitive Function, Neuropsychology, Attention, Executive Function.

Keywords plus

Mothers, Psychology, Neuropsychological Tests, Executive Function.
} 
Los individuos, al interactuar constantemente con un entorno natural y social, se ven expuestos a diferentes situaciones y factores que conllevan a modificaciones del organismo, para facilitar su adaptación y ajuste; sin embargo, algunas de estas respuestas pueden producir efectos adversos, particularmente cuando se trata del estrés.

El estrés se considera como aquella respuesta del organismo ante la identificación de condiciones adversas o amenazantes (internas o externas) contra la homeostasis (Benitzhak \& Verny, 2004; Valencia-Alfonso, Feria-Velasco, Luquín, DíazBurke \& García-Estrada, 2004). El estrés implica la sobreactivación de varios sistemas, principalmente del eje hipotálamo-hipófisis-adrenal, y la consiguiente liberación de la Hormona Liberadora de la Corticotropina en el hipotálamo, su acción sobre la hipófisis y la segregación de la Hormona Adenocorticotrópica (ACTH), que ejerce efecto por vía sanguínea sobre las glándulas adrenales, consiguiendo la liberación de las hormonas corticoesteroideas, ya sean glucocorticoides (GC), mineralocorticoides (MC), u hormonas sexuales, que tendrán un efecto sistémico (Valencia-Alfonso et al., 2004), que puede producir cambios a largo plazo.

Diversos estudios han señalado que estos efectos del estrés no se reducen únicamente a quien experimenta el estresor, sino que también pueden trascender y afectar el desarrollo del feto y de las crías, cuando lo experimenta una madre gestante. Mulder, Robles de Medina, Huizink, Van den Bergh, Buitelaar y Visser, en el 2002 (citados por Benitzhak \& Verny, 2004), describen 3 mecanismos que pueden operar simultáneamente para explicar la forma en que la señal de estrés materno puede alcanzar al feto. Los posibles mecanismos son: reducción del flujo sanguíneo del feto durante el incremento de los niveles de estrés materno, transporte transplacentario de las hormonas maternas y liberación de CRH placentaria inducida por el estrés al ambiente intrauterino.

Estudios en animales han mostrado que el estrés materno puede producir cambios en las crías que en algunos casos son irreversibles y que se manifiestan en la alteración de la distribución de diferentes tipos de neurotransmisores (noradrenalina y dopamina), disminución o incremento del cuerpo calloso (Benitzhak \& Verny, 2004), retrasos en el desarrollo neuromotor, disminución de la conducta exploratoria y deterioro de la adaptación a las condiciones conflictivas (Clarke et al., 1993; Weinstock et al., 1992; Takahashi et al. 1990 citados por Huizink et al., 2002), lo cual sugiere que existe una gran susceptibilidad al estrés materno durante la vida fetal.

Por su parte, los estudios en seres humanos han indicado que el estrés materno prenatal está relacionado con incremento en los abortos espontáneos, mayor inquietud en los fetos durante el ultrasonido, y mayor irritabilidad, llanto y dificultad para calmarse en los primeros meses de vida (Piontelli, 1992 y Van den Berg, 1990 citados por Benitzhak \& Verny, 2004; Grizenko, Shayan, Polotskaia, Ter-Stepanian \& Joober, 2008).). También se ha descrito que la experimentación de estrés por parte de la madre, en situaciones que percibe como aversivas o amenazantes, se correlaciona con trastornos en los infantes, los cuales se pueden manifestar en malformaciones congénitas, disminución del peso al nacer , menor tiempo de gestación, alteraciones neuroendocrinas y del neurodesarrollo motor y sensorial, trastornos psiquiátricos como esquizofrenia y trastornos de conducta (Benitzhak \& Verny, 2004; Foster, 2006; Grizenko et al., 2008; Hougaard, Andersen, Kjær, Hansen, Werge \& Lund, 2005; Torstensson, Lundberg, Sveger \& McNeil, 1999). Además, existen estudios que han asociado dificultades cotidianas en los primeros meses del embarazo, con problemas cognitivos en los hijos, manifestándose en problemas de atención y puntuaciones más bajas en pruebas de capacidad intelectual (Huizink, De Medina, Mulder, Visser \& Buitelaar, 2002 en Benitzhak \& Verny, 2004).

Una madre gestante, además de verse expuesta a las situaciones que puede enfrentar cualquier individuo, también puede vivenciar otros eventos que se asocian directamente con las condiciones del embarazo. Entre éstos, encontramos los factores que se describen a continuación en la Tabla 1. 
TABLA 1

Tipos de estresores vivenciados por mujeres durante la gestación

\begin{tabular}{|c|c|}
\hline $\begin{array}{l}\text { Estresores psicosociales } \\
\text { generales o permanentes }\end{array}$ & $\begin{array}{l}\text { Estresores relacionados } \\
\text { con la gestación }\end{array}$ \\
\hline $\begin{array}{l}\text { Estrés laboral } \\
\text { Dificultades económicas } \\
\text { Relación marital } \\
\text { Estrés psicosocial: racismo, } \\
\text { discriminación, violencia } \\
\text { personal } \\
\text { Estrés agudo ocasionado por } \\
\text { eventos externos: guerras, } \\
\text { terrorismo, desastres natura- } \\
\text { les, etc. }\end{array}$ & $\begin{array}{l}\text { Malestar físico (fatiga, } \\
\text { micciones frecuentes, } \\
\text { sudoración, dolor de } \\
\text { espalda) } \\
\text { Vulnerabilidad emocio- } \\
\text { nal causada por hormo- } \\
\text { nas y miedo (al parto, } \\
\text { a la paternidad y por la } \\
\text { salud del bebé) }\end{array}$ \\
\hline
\end{tabular}

Factores de vulnerabilidad

Edad: extremos de la vida

Poca experiencia previa o entrenamiento relevante en el afrontamiento a desastres

Minoría étnica

Estatus socioeconómico bajo

Exposición severa al desastre, especialmente heridas, amenazas a la vida y pérdidas extremas

Vivir en comunidades perturbadas o traumatizadas

Estrés secundario y pérdida de recursos materiales o sociales

Fuente: elaboración propia con base en Benitzhak y Verny (2004).

Para el caso específico de la población estudiada, se debe tener en cuenta que Colombia es un país en vías de desarrollo, que simultáneamente presenta un conflicto interno generador de diversas situaciones que pueden llevar a producir estrés en la población en general. Para las mujeres, existen condiciones de desigualdad y de vulnerabilidad que se manifiestan en aspectos económicos (menor remuneración laboral y difícil acceso al empleo), sociales (violencia intrafamiliar), de salubridad (precariedad en la atención, mortalidad materna, embarazos no deseados, embarazos de alto riesgo y embarazo adolescente), políticos y culturales (PNUD, 2006). Para la población bogotana se siguen presentando estas mismas condiciones, aunque con una disminución importante respecto al resto del país.
De esta manera, considerando los diversos factores biológicos, psicológicos y sociales de nuestra población y las posibles respuestas de estrés mencionados anteriormente, el presente estudio busca describir dentro de una población clínica de la ciudad de Bogotá, las características cognitivas de niños, niñas, y adolescentes, cuyas madres reportan estrés prenatal, como único factor de riesgo asociado.

\section{Método}

Se registraron todos los casos evaluados en el Servicio de Neuropsicología de Consultores en Psicología de la Pontificia Universidad Javeriana de Bogotá-Colombia, entre los años 2007 y 2009. Todas las madres participaron en un protocolo de entrevista, donde se incluía información sobre el desarrollo pre, peri y postnatal de los menores, incluido el reporte y descripción de las posibles situaciones de estrés experimentadas por las mujeres, durante la gestación. Todas las madres firmaron un consentimiento informado en el que autorizaban el uso de esta información con fines investigativos. A cada caso se le asignó un código, para proteger la identidad de los pacientes y sus familias.

Se revisaron en total 247 historias, de las cuales se descartaron aquellos casos en donde los y las participantes tuvieran condiciones que se consideran riesgos para el adecuado desarrollo de sus habilidades cognoscitivas (enfermedades maternas durante la gestación o previa, entre éstas: toxoplasmosis, preeclampsia, diabetes, etc., y en los infantes condiciones genéticas manifiestas, enfermedad neurológica, hipoxias neonatales o perinatales, traumas físicos y/o emocionales, antecedentes personales psiquiátricos, entre otros). De esta manera, se seleccionaron aquellos sujetos que en su historia de desarrollo solo presentaban como factor de riesgo la vivencia de estrés por parte de la madre durante la gestación del paciente. Así, la muestra se conformó por un total de 31 menores.

A cada uno de los participantes se le aplicó un protocolo de evaluación cognitiva que incluía varias pruebas neuropsicológicas (Test de Aprendizaje Verbal España Complutense Infantil-AVECI, 
Evaluación neuropsicológica Infantil-ENI, Escala de Inteligencia de Kaufman para preescolar y primaria, K-ABC, WISC-IV), a partir del cual se realizó una caracterización de su desempeño cognitivo global, que permitió la construcción de los perfiles neuropsicológicos. Por tratarse de una población clínica, se utilizó un protocolo estándar, con variaciones de acuerdo al motivo de consulta y que se ajustaba a las condiciones cronológicas y académicas de los participantes. No obstante, se empleó, como medida general, la Escala Wechsler de Inteligencia para Niños-WISC IV (Wechsler, 2005). Posteriormente, se hizo un análisis descriptivo de las características de la población y su relación con el estrés materno durante el embarazo.

\section{Resultados}

El grupo seleccionado corresponde al 12,55\% de los casos atendidos, entre los años 2007 y 2009. Todos los participantes residen en la ciudad de Bogotá; presentan como edad promedio 9,7 años, con un rango de edades comprendido entre los 6 a 15 años. Cuatro menores son de género femenino $(12,9 \%)$ y 27 son de género masculino $(87,1 \%)$. La dominancia manual se orienta hacia preferencia derecha $(93,54 \%$ de la muestra), mientras que la lateralidad izquierda $(3,2 \%)$ y no definida $(3,2 \%)$ representan la minoría. El mayor porcentaje de menores se encontraba cursando la Educación Básica Primaria, al momento de la valoración. Estos datos se resumen en la Tabla 2.

De acuerdo con el reporte de las madres, se clasificaron los factores que producen estrés en las categorías expuestas en la Tabla 3.

Se destaca que estas categorías surgen de los reportes maternos y no constituyen categorías excluyentes, pues las madres en varias ocasiones reportan más de un tipo de estresor, como por ejemplo, embarazo no deseado y situación económica.

Los participantes, al ser seleccionados de una población clínica, asisten al servicio Neuropsicológico, por diversos motivos de consulta o remisión que se describen a continuación.

TABLA 2

Caracterización sociodemográfica de la muestra al momento de la evaluación

\begin{tabular}{|c|c|c|c|c|c|c|c|c|c|c|c|}
\hline \multicolumn{2}{|l|}{ Edad } & \multicolumn{4}{|c|}{ Género } & \multicolumn{2}{|c|}{$\begin{array}{c}\text { Dominancia } \\
\text { manual }\end{array}$} & \multicolumn{4}{|c|}{ Escolaridad } \\
\hline 6 años & 3 & $9,7 \%$ & Femenino & 4 & $12,9 \%$ & Diestros & 29 & $93,5 \%$ & Preescolar & 5 & $16 \%$ \\
\hline 7 años & 5 & $16 \%$ & Masculino & 27 & $87,1 \%$ & Zurdos & 1 & $3,2 \%$ & Primaria & 19 & $61,3 \%$ \\
\hline 8 años & 3 & $9,7 \%$ & & & & No definida & 1 & $3,2 \%$ & Secundaria & 6 & $19,4 \%$ \\
\hline 9 años & 4 & $12,9 \%$ & & & & & & & Educación especial & 1 & $3,2 \%$ \\
\hline 10 años & 4 & $12,9 \%$ & & & & & & & & & \\
\hline 11 años & 4 & $12,9 \%$ & & & & & & & & & \\
\hline 12 años & 3 & $9,7 \%$ & & & & & & & & & \\
\hline 13 años & 1 & $3,2 \%$ & & & & & & & & & \\
\hline 14 años & 2 & $6,4 \%$ & & & & & & & & & \\
\hline 15 años & 2 & $6,4 \%$ & & & & & & & & & \\
\hline$N=$ & 31 & $100 \%$ & & & & & & & & & \\
\hline
\end{tabular}

Fuente: elaboración propia. 
TABla 3

Descripción de los factores asociados con estrés durante el periodo gestacional en la muestra estudiada

\begin{tabular}{ll}
\hline \multicolumn{1}{c}{ Categoría } & \multicolumn{1}{c}{ Descripción situaciones asociadas } \\
\hline Embarazo no deseado & $\begin{array}{l}\text { Producto de embarazos accidentales, deseos de no llevar la gestación } \\
\text { a término, afectación del proyecto vital de la mujer y su familia. }\end{array}$ \\
$\begin{array}{l}\text { Estrés laboral } \\
\text { Estrés por abandiciones laborales de alta demanda física o acoso laboral. } \\
\text { con la condición de madre soltera. }\end{array}$ & No contar con el progenitor como protector o proveedor. \\
Situación económica & Pérdida del empleo o no contar con apoyo económico. \\
Mala relación de pareja & Maltrato por parte del compañero o conflictos maritales. \\
Conflictos familiares & Malas relaciones con la familia, rechazo o maltrato intrafamiliar. \\
Complicaciones o temores relacionados con & Por antecedentes como abortos o pérdidas previas. \\
el embarazo & \\
Duelo y/o depresión & $\begin{array}{l}\text { Pérdida del compañero o familiar significativo durante la gestación. } \\
\text { Tristeza considerable por las situaciones relacionadas con las catego- } \\
\text { rías previas. }\end{array}$ \\
\hline
\end{tabular}

Fuente: elaboración propia.

TABLA 4

Motivos de consulta/ remisión de los participantes al acudir al servicio de Neuropsicología

\begin{tabular}{|c|c|c|c|c|c|}
\hline \multicolumn{6}{|c|}{ Motivos de Consulta y/o remisión (MC/R) } \\
\hline Sujeto \# & $\begin{array}{c}\text { Problemas de } \\
\text { atención }\end{array}$ & $\begin{array}{c}\text { Problemas de } \\
\text { comportamiento }\end{array}$ & $\begin{array}{c}\text { Dificultades } \\
\text { de aprendizaje }\end{array}$ & $\begin{array}{c}\text { Determinar el } \\
\text { coeficiente intelectual }\end{array}$ & Otros (lenguaje) \\
\hline 1 & & & $\mathrm{X}$ & $\mathrm{X}$ & \\
\hline 2 & & $\mathrm{X}$ & & & \\
\hline 3 & & $\mathrm{X}$ & $\mathrm{X}$ & & \\
\hline 4 & & & $\mathrm{X}$ & & \\
\hline 5 & & & $\mathrm{X}$ & & \\
\hline 6 & & & $\mathrm{X}$ & & \\
\hline 7 & $\mathrm{X}$ & & & & \\
\hline 8 & $\mathrm{X}$ & $\mathrm{X}$ & & $\mathrm{X}$ & \\
\hline 9 & & & & & $\mathrm{X}$ \\
\hline 10 & $\mathrm{X}$ & $\mathrm{X}$ & & & \\
\hline 11 & & & & $\mathrm{X}$ & \\
\hline 12 & & $\mathrm{X}$ & $\mathrm{X}$ & & \\
\hline 13 & $\mathrm{X}$ & $\mathrm{X}$ & & & \\
\hline 14 & $\mathrm{X}$ & $\mathrm{X}$ & & & \\
\hline
\end{tabular}




\begin{tabular}{|c|c|c|c|c|c|}
\hline \multicolumn{6}{|c|}{ Motivos de Consulta y/o remisión (MC/R) } \\
\hline Sujeto \# & $\begin{array}{c}\text { Problemas de } \\
\text { atención }\end{array}$ & $\begin{array}{c}\text { Problemas de } \\
\text { comportamiento }\end{array}$ & $\begin{array}{c}\text { Dificultades } \\
\text { de aprendizaje }\end{array}$ & $\begin{array}{c}\text { Determinar el } \\
\text { coeficiente intelectual }\end{array}$ & Otros (lenguaje) \\
\hline 15 & $\mathrm{X}$ & & & & \\
\hline 16 & $\mathrm{X}$ & $\mathrm{X}$ & & & \\
\hline 17 & & & $\mathrm{X}$ & & \\
\hline 18 & & & $\mathrm{X}$ & $\mathrm{X}$ & \\
\hline 19 & & & $\mathrm{X}$ & $\mathrm{X}$ & \\
\hline 20 & $\mathrm{X}$ & & & & \\
\hline 21 & & & & $\mathrm{X}$ & \\
\hline 22 & & & $\mathrm{X}$ & & \\
\hline 23 & $\mathrm{X}$ & $\mathrm{X}$ & & & \\
\hline 24 & & & & $\mathrm{X}$ & \\
\hline 25 & $\mathrm{X}$ & $\mathrm{X}$ & & & \\
\hline 26 & & & & $\mathrm{X}$ & \\
\hline 27 & & & $X$ & & \\
\hline 28 & $X$ & & $X$ & $\mathrm{X}$ & \\
\hline 29 & $\mathrm{X}$ & $\mathrm{X}$ & $\mathrm{X}$ & & \\
\hline 30 & & & $\mathrm{X}$ & & \\
\hline \multicolumn{6}{|l|}{31} \\
\hline Mc único & 3 & 1 & 7 & 4 & 2 \\
\hline Mc combinado & 12 & 11 & 14 & 9 & 2 \\
\hline \multicolumn{6}{|c|}{ Motivos de consulta combinados } \\
\hline Atención/cpto & Atención/ci & Atención/apzje & Cpto/apzje & Apzje/ci & \\
\hline 7 & 1 & 1 & 2 & 4 & \\
\hline
\end{tabular}

CPTO: comportamiento

APZJE: aprendizaje

CI: valoración para determinar el Coeficiente Intelectual

Fuente: elaboración propia.

De los 31 sujetos, se encontró que 17 (54.84\%) tenían un único motivo de consulta. De este grupo, el motivo de consulta más común: fue dificultades de aprendizaje y bajo rendimiento académico. Asimismo, el grupo restante, conformado por 14 pacientes, $(45,16 \%)$ tenía múltiples motivos de consulta. En este grupo, la combinación más frecuente fueron los problemas de atención y de comportamiento, en 7 de los casos.

Del funcionamiento cognitivo, se analizaron las puntuaciones en las escalas de Inteligencia WISC IV, identificándose, por promedio, las escalas donde se obtuvieron menores desempeños, y considerando como puntuaciones escalares sig- 
nificativas aquellas que fueran iguales o menores a 7 (véase Tabla 5).

Se observa que los mayores porcentajes de sujetos presentan puntuaciones que se encuentran hacia el extremo inferior de los valores escalares esperados.

Como se observa en la Tabla 6, los desempeños de los participantes muestran una tendencia a ubi- carse dentro de los extremos bajos de la puntuación escalar esperada (alrededor de 7).

$\mathrm{Al}$ analizar el Coeficiente Intelectual, se advierte una tendencia similar a la descrita anteriormente con las subescalas, principalmente para el CI Total Promedio $(83,5)$ y el índice de Velocidad de Procesamiento, pues los puntajes oscilan entre el promedio normal $(85-115)$ y el límite bajo de este rango (70-85), como se observa en la Tabla 7.

TABLA 5

Subescalas del WISC IV donde se encontraron los menores desempeños

\begin{tabular}{cccccc}
\hline \multicolumn{5}{c}{ Subescalas del WISC IV } \\
\hline Puntuación Escalar & $\begin{array}{c}\text { Retención de } \\
\text { dígitos }\end{array}$ & Matrices & Comprensión & $\begin{array}{c}\text { Búsqueda de } \\
\text { símbolos }\end{array}$ & Registros \\
$>13$ & $50 \%$ & $38,1 \%$ & $45,5 \%$ & $48 \%$ & $45,5 \%$ \\
$7-13$ & $50 \%$ & $52,4 \%$ & $54,5 \%$ & $52 \%$ & $54,5 \%$ \\
$<7$ & 6,9 & 6,8 & 6,0 & 6,5 & 6,1 \\
\hline Puntuación escalar Promedio & & & & & \\
\hline
\end{tabular}

Fuente: elaboración propia.

TABLA 6

Puntuaciones promedio de las subescalas del WISC IV donde se identificaron desempeños dentro del rango normal

\begin{tabular}{ccccc}
\hline \multicolumn{5}{c}{ Subescalas del WISC IV } \\
\hline Diseño con Cubos & Semejanzas & Conceptos con dibujos & Claves & Vocabulario \\
8,3 & 7,1 & 8,0 & 7,5 & 7,6 \\
\hline \multicolumn{5}{c}{ Subescalas del WISC IV } \\
\hline Letras y números & Figuras Incompletas & Información & Aritmética & Razonamiento con pistas \\
8,1 & 8,4 & 7,2 & 7,7 & 10,5 \\
\hline
\end{tabular}

Fuente: elaboración propia.

\section{TABLA 7}

Distribución de los porcentajes de funcionamiento intelectual global y los índices de funcionamiento para la escala WISC IV

\begin{tabular}{cccccc}
\hline \multirow{2}{*}{ Rango de puntuaciones } & $\begin{array}{c}\text { Coeficiente inte- Índice de Com- Índice de Razona- Índice de memo- Índice de Velocidad } \\
\text { lectual Global }\end{array}$ & $\begin{array}{c}\text { prensión verbal } \\
\text { miento perceptual }\end{array}$ & $\begin{array}{c}\text { ria de trabajo } \\
\text { de procesamiento }\end{array}$ \\
\hline $85-115$ & $48,4 \%$ & $45 \%$ & $50 \%$ & $55 \%$ & $35 \%$ \\
$70-84$ & $32,3 \%$ & $45 \%$ & $30 \%$ & $35 \%$ & $35 \%$ \\
$<70$ & $19,35 \%$ & $10 \%$ & $20 \%$ & $10 \%$ & $30 \%$ \\
\hline Puntuación promedio & 83,5 & 85 & 86 & 85,9 & 79,7 \\
\hline
\end{tabular}

Fuente: elaboración propia. 
Partiendo de los hallazgos cuantitativos, se analiza caso por caso, y de acuerdo con los desempeños, ejecuciones y al análisis clínico/ conceptual de los evaluadores, se elaboraron perfiles neuropsicológicos, basados en las áreas de funcionamiento cognoscitivo que manifiestan déficits o dificultades.

Según la Tabla 8, la habilidad cognitiva que presenta un mayor compromiso corresponde a las funciones ejecutivas, dentro de las cuales sobresalen los problemas de memoria de trabajo; le siguen la atención y la velocidad de procesamiento. Es de resaltar que, en la mayoría de los casos, se encon- tró la tríada: dificultades de atención, funciones ejecutivas y velocidad de procesamiento.

Este hecho corresponde a lo descrito en los índices y escalas del WISC IV, donde se observan como menor índice, el de Velocidad de Procesamiento de la información, y fallas en las subescalas que se relacionan, justamente, con la atención (Registros, Búsqueda de Símbolos) y Función ejecutiva (Matrices, Comprensión y Retención de dígitos: Índice de memoria de trabajo).

Discusión

En el presente estudio se buscó describir las características cognitivas halladas en menores

TABLA 8

Dificultades cognoscitivas encontradas en la evaluación neuropsicológica

\begin{tabular}{|c|c|c|c|c|c|c|c|c|c|c|c|}
\hline \multirow[b]{2}{*}{ Sujeto \# } & \multicolumn{11}{|c|}{ Funciones Cerebrales superiores } \\
\hline & AT & VP & $\mathrm{FE}$ & Leng Exp & Leng Comp & Lecto / Escr & MemoVerb & $\begin{array}{l}\text { Memo- } \\
\text { Visual }\end{array}$ & Prax. & $\begin{array}{l}\text { Hab. } \\
\text { Espa }\end{array}$ & $\begin{array}{c}\text { Cálculo } \\
\text { Arit }\end{array}$ \\
\hline 1 & $X$ & $X$ & $\mathrm{X}$ & & & $\mathrm{X}$ & & & & & \\
\hline 2 & & $\mathrm{X}$ & $\mathrm{X}$ & & & & & & & & \\
\hline 3 & $\mathrm{X}$ & & $\mathrm{X}$ & $\mathrm{X}$ & & $\mathrm{X}$ & & $\mathrm{X}$ & $\mathrm{X}$ & & $\mathrm{X}$ \\
\hline 4 & $\mathrm{X}$ & $\mathrm{X}$ & $\mathrm{X}$ & & & & & & & & \\
\hline 5 & $\mathrm{X}$ & $\mathrm{X}$ & & & & & & & & & \\
\hline 6 & & & & & & $\mathrm{X}$ & & & & & \\
\hline 7 & & $\mathrm{X}$ & $\mathrm{X}$ & $\mathrm{X}$ & & & & & $\mathrm{X}$ & $\mathrm{X}$ & \\
\hline 8 & $\mathrm{X}$ & $\mathrm{X}$ & $\mathrm{X}$ & & & & & & & & \\
\hline 9 & & & $\mathrm{X}$ & & & & & & & & \\
\hline 10 & $\mathrm{X}$ & & $\mathrm{X}$ & & & & $\mathrm{X}$ & & & & $\mathrm{X}$ \\
\hline 11 & $\mathrm{X}$ & $\mathrm{X}$ & $\mathrm{X}$ & & $\mathrm{X}$ & & & & & $\mathrm{X}$ & \\
\hline 12 & $\mathrm{X}$ & & & & & $\mathrm{X}$ & & & & & \\
\hline 13 & & & & & & & & & & $\mathrm{X}$ & \\
\hline 14 & & & $\mathrm{X}$ & & & & & & & & \\
\hline 15 & & & & $\mathrm{X}$ & & & & & & & \\
\hline 16 & & $\mathrm{X}$ & X & & & & & & & & \\
\hline 17 & $X$ & $\mathrm{X}$ & X & & & & & & & & \\
\hline 18 & $X$ & $X$ & $X$ & & & & & & & & \\
\hline 19 & & & X & & & & & & & & \\
\hline 756 & I $\mathrm{L}$ & UnIVE & RSITA & As PsYcho & DLOGICA | & \begin{tabular}{l|l} 
V. 9 & $\mathrm{No}$
\end{tabular} & $.3 \mid$ SEPTI & IEMBRE-D & DICIEMP & BRE | & 2010 \\
\hline
\end{tabular}




\begin{tabular}{|c|c|c|c|c|c|c|c|c|c|c|c|}
\hline \multirow[b]{2}{*}{ Sujeto \# } & \multicolumn{11}{|c|}{ Funciones Cerebrales superiores } \\
\hline & AT & VP & $\mathrm{FE}$ & Leng Exp & Leng Comp & Lecto / Escr & MemoVerb & $\begin{array}{l}\text { Memo- } \\
\text { Visual }\end{array}$ & Prax. & $\begin{array}{l}\text { Hab. } \\
\text { Espa }\end{array}$ & $\begin{array}{c}\text { Cálculo } \\
\text { Arit }\end{array}$ \\
\hline 20 & $\mathrm{X}$ & $\mathrm{X}$ & $\mathrm{X}$ & $\mathrm{X}$ & & $\mathrm{X}$ & & & & & \\
\hline 21 & & $\mathrm{X}$ & $\mathrm{X}$ & & & & & & & & \\
\hline 22 & $\mathrm{X}$ & $\mathrm{X}$ & $\mathrm{X}$ & & & $\mathrm{X}$ & & & $\mathrm{X}$ & & $\mathrm{X}$ \\
\hline 23 & & & $\mathrm{X}$ & & & & & & & & \\
\hline 24 & $\mathrm{X}$ & & $\mathrm{X}$ & $\mathrm{X}$ & & & $\mathrm{X}$ & & & & \\
\hline 25 & $\mathrm{X}$ & & & & & & & & & & \\
\hline 26 & $\mathrm{X}$ & $\mathrm{X}$ & $\mathrm{X}$ & & & & & & & & \\
\hline 27 & $\mathrm{X}$ & $\mathrm{X}$ & $\mathrm{X}$ & & & $\mathrm{X}$ & & & & & $\mathrm{X}$ \\
\hline 28 & $X$ & & X & & & & & & & & \\
\hline 29 & & & $\mathrm{X}$ & & & & & & & & \\
\hline 30 & & & $\mathrm{X}$ & & & $\mathrm{X}$ & & & & & \\
\hline 31 & $\mathrm{X}$ & X & & & & & & & & & \\
\hline $\begin{array}{l}\text { Frecuencia de } \\
\text { presentación de } \\
\text { la dificultad }\end{array}$ & 18 & 16 & 24 & 5 & 1 & 8 & 2 & 1 & 3 & 3 & 4 \\
\hline
\end{tabular}

AT: atención, VP: Velocidad de Procesamiento, FE: Funciones ejecutivas, Leng. Exp.: Lenguaje expresivo, Leng. Comp: Lenguaje Comprensivo, Lecto/Escr: Lecto/escritura, Memo Verb: Memoria Verbal, Memo Visual: Memoria Visual, Prax: Praxias, Hab. Espa; Habilidades Espaciales, Cálculo/Aritmética.

Fuente: elaboración propia.

residentes en Bogotá-Colombia, que asistieron al servicio de neuropsicología clínica y cuyas madres reportaron haber padecido estrés durante el embarazo, como único posible factor de riesgo cognitivo prenatal de sus hijos.

Los motivos de consulta se relacionaban principalmente con problemas de rendimiento académico, de atención y de comportamiento, siendo éstas las quejas primordiales, por parte de los educadores, de los profesionales de la salud, así como de los propios padres.

Desde una postura clínica, los motivos de remisión y las descripciones sobre el funcionamiento de los niños y niñas, sugieren características semiológicas similares a las descritas en el Trastorno por Déficit de Atención e Hiperactividad (TDAH tipo combinado) con una alta disfunción a nivel escolar (problemas de aprendizaje). En este aspecto, auto- res como Grizenko et al. (2008) han señalado que existe una relación entre la presencia de estrés moderado durante la gestación, y mayor severidad de los síntomas del TDAH. En el caso de este estudio, los reportes hechos por las madres indican vivencia de estrés moderado durante todo el proceso de gestación, como se describió en la Tabla 3. Estos estresores son moderados, porque no existían factores que potenciarán el riesgo ni representaban un malestar significativo, que condujera a las madres a buscar alguna clase de apoyo social o asistencial, como suele ocurrir en situaciones de estrés agudo y/o severo (véanse Grizenko et al., 2008).

En relación con el reporte subjetivo de la madre alrededor de los tipos de estresores y la dinámica de afectación de éstos, se debe resaltar que no existe un único factor experimentado por estas mujeres y que ninguno de los descritos se relaciona con un 
motivo de consulta específico. Por el contrario, se observa que los estresores son múltiples y pueden aparecer de manera simultánea o secuencial a lo largo de la gestación, siendo unos, en algunos casos, resultado indirecto de los otros, como se ve, por ejemplo, cuando existen problemas en el acceso a recursos económicos, que llevan a manifestar conflictos en las relaciones maritales.

En el grupo de participantes estudiado, se consiguió determinar un perfil neuropsicológico que se caracteriza por mostrar tendencias hacia fallos específicos en el desempeño cognitivo.

En primera instancia, se observó que las capacidades cognoscitivas donde se encuentran compromisos (véase Tabla 8), son las funciones ejecutivas, la atención y la velocidad de procesamiento.

Dentro de las funciones ejecutivas, los problemas se ubican principalmente en la memoria de trabajo, la abstracción, la categorización verbal, el juicio social y la inhibición cognitiva y conductual. Estos hallazgos correlacionan con puntuaciones por debajo de lo esperado (puntuaciones escalares $\leq 7)$ en los subtests Retención de Dígitos, Matrices, Comprensión y Semejanzas de la escala WISC IV (véanse Tablas 5 y 6). En atención y velocidad de procesamiento, se encontraron desempeños deficientes en tareas de rastreo visual y atención selectiva que se observan en los subtests Búsqueda de Símbolos y Registros y, a partir de éstos, en el Promedio del Índice de Velocidad de Procesamiento (IVP $=79.7$ ).

Estas características cognitivas se presentan en conjunto como una triada clínica que afecta el funcionamiento de los niños y niñas, en su rendimiento en actividades escolares y en el comportamiento.

Estas esferas cognitivas al ser primordiales para el adecuado desarrollo y funcionamiento de las demás habilidades cognitivas y escolares, repercuten sobre el funcionamiento intelectual general, que demostró una tendencia que oscila entre desempeños normales bajos y limítrofes. Esto se confirma al evidenciar el porcentaje de sujetos que obtienen un capacidad intelectual general (CI) en los rangos menores a 100 (Promedio Puntuación Total CI $=83,5$ Tabla 7), coincidiendo con lo descrito, en 2002, por Huizink et al. (citado por Benitzhak \&
Verny, 2004) cuando se refiere a que hijos de mujeres expuestas a estrés durante los primeros meses del embarazo, presentaban puntuaciones más bajas en pruebas de capacidad intelectual.

Los hallazgos referidos, son sugerentes de una posible disfunción en circuitos cerebrales prefrontales y sus conexiones subcorticales, sistemas que son regulados por vías catecolaminérgicas, hipótesis de un posible efecto de las respuestas del estrés sobre estos sistemas neurales, como se han descrito en modelos animales (Benitzhak \& Verny, 2004). Se ha descrito también la posibilidad de que exista algún tipo de sensibilidad directa ante experiencias adversas sobre la Corteza Prefrontal Medial, lo cual resulta en atrofia celular y reducción dendrítica en estudios con ratas (Elzinga \& Bremner, 2002; Pascual, Zamora \& Valero, 2006,). De igual manera se ha reportado que las posibles alteraciones de la Corteza Prefrontal Medial se relacionan con alteraciones en la transmisión del GABA (neurotransmisor inhibidor) (Pascual et al., 2006). Al considerarse el papel de la corteza prefrontal y la regulación que ejerce sobre el control inhibitorio tanto cognitivo como conductual, a través de los circuitos gabaérgicos, y las alteraciones evidenciadas en las funciones ejecutivas y atencionales en los hijos de mujeres que sufrieron estrés durante la gestación, se puede plantear la posibilidad de considerar que los efectos fisiológicos, conductuales y cognitivos descritos ante condiciones de estrés materno prenatal, pueden tener una explicación neurofisiológica similar a la de los compromisos cognitivos en los casos de estrés postnatal.

Así mismo, se puede considerar un efecto diferencial en la vulnerabilidad al estrés prenatal de acuerdo al sexo, como describen León y Dueñas (2009), en el caso de estrés por separación materna temprana, donde se identificó reducción de los receptores Gaba A en machos pero no en hembras, que podría relacionarse con el hallazgo del presente estudio, donde se encuentra una diferencia considerable en el porcentaje de niños $(87,1 \%)$ que consultan, sobre las niñas (12,9\%), indicando que posiblemente exista una incidencia y vulnerabilidad diferencial con una mayor expresión de déficits cognitivos en varones, aunque la exposición 
al estrés se presente de igual manera en madres gestantes sin distinción por el sexo de sus hijos.

Se dejan abiertas, entonces, varias posibilidades de investigación, con el objeto de confirmar los supuestos aquí planteados, a través de estudios correlacionales con control de variables, el uso de modelos animales para desarrollar explicaciones neurofuncionales y el seguimiento de muestras clínicas de mayor tamaño con grupos de comparación, donde se puedan contrastar los perfiles de desempeño cognoscitivo asociados con otras condiciones de riesgo pre, peri y postnatal, además de la interacción entre estos factores (por ejemplo la posible relación entre el estrés materno y el desarrollo de preeclampsia). Finalmente, el objeto de este tipo de investigaciones está orientado a confirmar la interacción de múltiples factores sociales, biológicos, cognitivos y emocionales, como fuente de explicación de las condiciones clínicas que afectan a los niños y niñas y, aún más, a la consideración de la sintonía que existe entre el ambiente materno (genético, social) y el desarrollo infantil, orientado a la generación de programas de promoción y prevención de salud, particularmente en comunidades como la colombiana.

\section{Referencias}

Benitzhak, Y. \& Verny T. R. (2004). The nature of stress due to terrorism on pregnant women and their offspring. Journal of Prenatal EO Perinatal Psychology EO Health, 19 (1), p. 65-74.

Elzinga, B. M. \& Bremner, J. D. (2002). Are the neural substrates of memory the final common pathway in posttraumatic stress disorder (PTSD)? Journal of Affective Disorders, 70 (1), 1-17.

Foster, S. (2006). A literature review: The effects of maternal stress in pregnancy on sensory integration in children. Journal of Prenatal \& Perinatal Psychology Eु Health, 21 (1), 83-91.

Grizenko, N., Shayan, Y. R., Polotskaia, A., Ter-Stepanian, M. \& Joober, R. (2008). Relation of maternal stress during pregnancy to symptom severity and response to treatment in children with ADHD. Journal of Psychiatry $\& \mathcal{O}$ Neuroscience, 33 (1), 10-6. Hougaard, K. S., Andersen, K., Hansen, W. \& Lund, S. P. (2005). Prenatal stress may increase vulnerability to life events: Comparison with the effects of prenatal dexamethasone. Developmental Brain Research, 159, 55-63.

Huizink, A. C., De Medina, P. R., Mulder, E. J. H., Visser, G. H. A. \& Buitelaar, J. K. (2002). Prenatal maternal stress, HPA axis activity, and postnatal infant development. International Congress Series, 1241, 65-71.

León, R., Diego, A. \& Dueñas, Z. J. (2009, diciembre). El estrés inducido por separación maternal temprana modifica en ratas adultas la expresión cerebral de receptores GABA A. Memorias del Encuentro Nacional de Investigadores en Postgrados, Universidad Nacional de Colombia.

Pascual, R., Zamora-León S. P. \& Valero-Cabré, A. (2006). Effects of postweaning social isolation and re-socialization on the expression of Vasoactive Intestinal Peptide (VIP) and dendritic development in the medial prefrontal cortex of the rat. Acta Neurobiologiæ Experementalis, 66, 7-14.

Programa de Naciones Unidas para el Desarrollo \& Departamento Nacional de Planeación. (2006). Informe de Colombia para los Objetivos de Desarrollo del Milenio. Hacia una Colombia equitativa e incluyente. Bogotá: Autores.

Torstensson, G., Lundberg, L., Sveger, T. \& McNeil, T. F. (1999). Maternal distress and congenital malformations: Do mothers of malformed fetuses have more problems? Journal of Psychiatric Research, 33, 291-301.

Valencia-Alfonso, C. E., Feria-Velasco, A., Luquín, S., Díaz-Burke, Y. \& García-Estrada, J. (2004). Efectos cerebrales del medio ambiente social. Revista de Neurología, 38 (9), 869-878.

Wechsler, D. (2005). Escala Wechsler de Inteligencia para Niños WISC IV. México: Manual Moderno. 
